\title{
Inbetriebnahme einer Präzisions-Apparatur für Wärme- übergangsmessungen beim Sieden reiner Stoffe und Nachrüstung für Stoffgemische / Teil II
}

\author{
Josef Schmadl, Peter Ignaszewski, Jens-Uwe Gerking
}

\section{Einleitung}

In Teil I der gleichnamigen Arbeit [1] waren die Inbetriebnahme der Apparatur und Testmessungen mit reinen Stoffen behandelt worden. Dieser Teil II bezieht sich im wesentlichen auf die Nachrüstung für Stoffgemische und Kontrollmessungen mit Gemischen.

\section{Apparative Ergänzungen}

Die Nachrüstung für Stoffgemische wurde durch Einbau von Probennahmeschleusen erreicht. Außerdem wurde die Meßwerterfassung, -auswertung und -archivierung weitgehend automatisiert durch Anbindung der Apparatur an ein vorhandenes Prozeßleitsystem.

\subsection{Probenahmeschleusen}

Zur Analyse von Gemischkonzentration und Phasengleichgewicht sind im Rahmen dieser Arbeit Probenahmestellen, zur Entnahme von flüssigem und dampfförmigem Versuchsstoff aus dem Verdampfer der Siedeapparatur, eingebaut worden (Abb.1). Die Proben werden über 1/8"-Rohrleitungen am Verdampfer, oben für die Dampfphase und unten für die Flüssigphase, entnommen und zwecks Analyse ausgeschleust. Die Schleusen, bestehen jeweils aus zwei Absperrventilen und einer dazwischenliegenden definierten Länge 6mm-Rohr. Am Ende der Schleuse vor dem zweiten Absperrventil ist ein Septum eingebaut um die Probe mit Hilfe einer Spritze passenden Volumens zu entnehmen. Jeweils hinter dem zweiten Absperrventil befindet sich ein Vakuumanschluß zum Spülen durch wiederholtes Entleeren und Evakuieren der Schleusen vor der Probenahme.

Die Flüssigkeitsprobenschleuse ist mit einem Rauminhalt von $2 \mathrm{ml}$ so ausgelegt, daß beim Befuillen in etwa das Volumen der Zuleitung aufgenommen wird. Durch mehrmaliges Spülen der Zuleitung können so Konzentrationsunterschiede zwischen Pool und Probe vermieden werden.

Die Dampfprobenahmeschleuse ist mit einem Rauminhalt von $10 \mathrm{ml}$ so ausgelegt, daß ungefähr das fünffache Zuleitungsvolumen aufgenommen wird. Außerdem wurde das Gefälle der Zuleitung so gewählt, daß „wildes" Kondensat in den Verdampfer zurïickläuft.

Trotzdem gab es Schwierigkeiten bei der dampfseitigen Probenahme, die im Zuge weiterer Entwicklungsarbeiten behoben werden sollen. So z.B. könnte eine Thermostatisierung der Dampfprobenschleuse zu einer Verbesserung der Probenqualität führen.

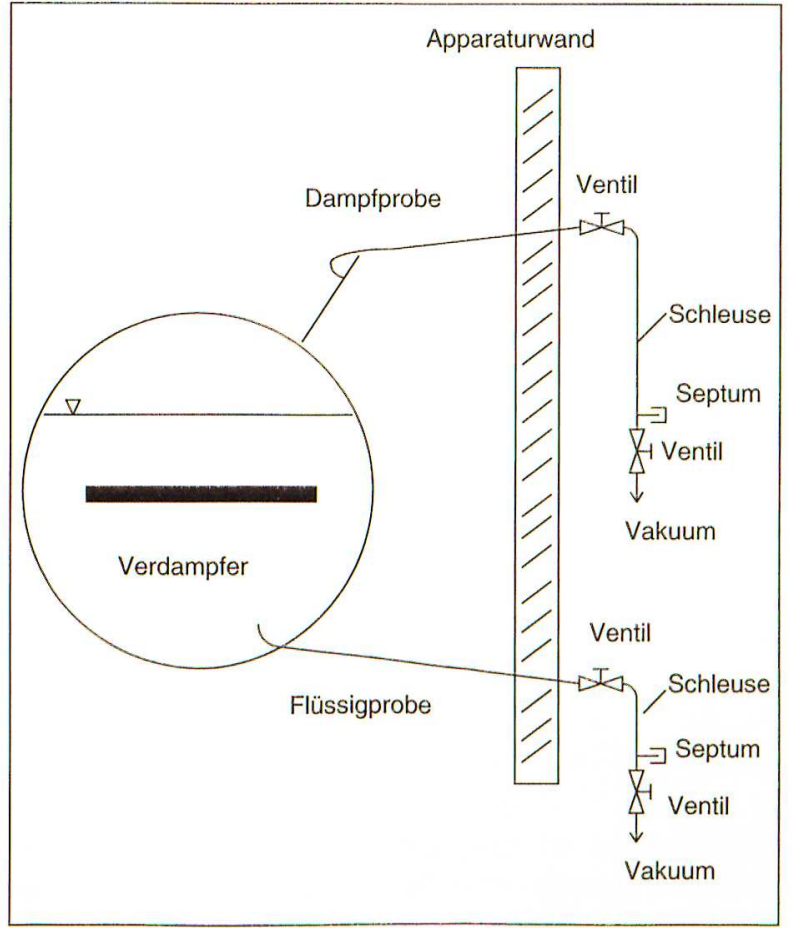

Abb.1: Schematische Darstellung der Probenahme aus Dampf- und Flüssigkeitsraum des Verdampfers

1.2 Anbindung an das Prozeßleitsystem und CIM/21 Im Rahmen dieser Arbeit erfolgte außerdem die Anbindung der Siedeapparatur an das Prozeßleitsystem (PLS) Contronic P zwecks Prozeßkontrolle durch Erfassen und Registrieren von Siededruck, Versuchsstoff-, Kühlkreislauftemperatur und Rohrwandüberhitzung. Um die Messdatenauswertung und -archivierung weitgehend zu automatisieren erfolgte eine Verknüpfung mit dem Prozeßdatenvisualisierungssystems CIM /21. Dies ermöglicht neben einer kontinuierlichen Meßwerteerfassung auch die Darstellung des dynamischen Verhaltens der Versuchsapparatur.

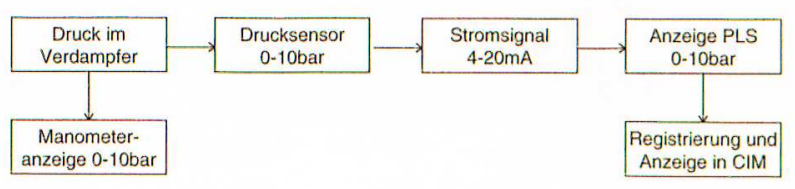

Abb. 2: Schematische Darstellung der Meßgröß3enerfassung anhand des Druckes

In Abb. 2 ist die Meßwerterfassung am Beispiel der Meßkette für den Druck dargestellt. Durch die Visualisierung ist es möglich die Meßdaten übersichtlich in einem erstellten Prozeßbild darzustellen (Abb. 3). 


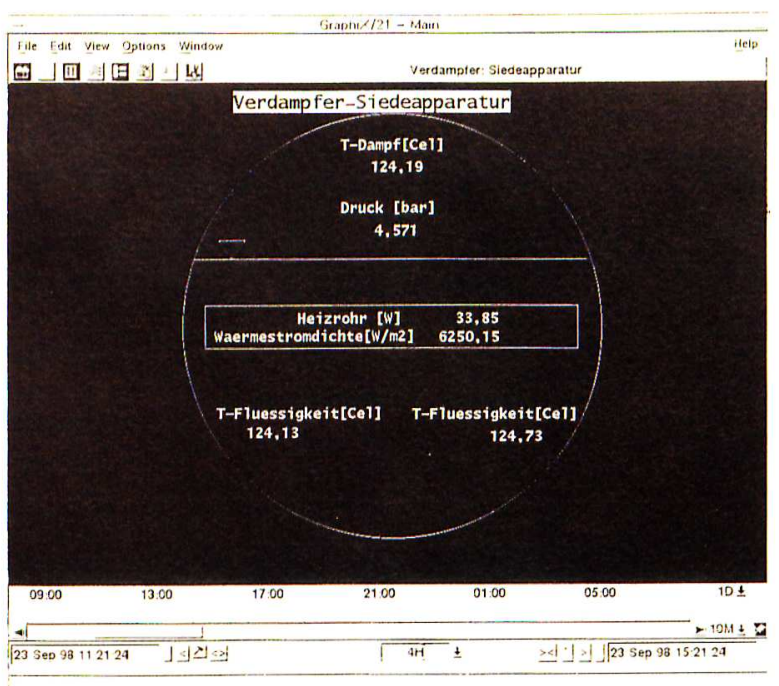

Abb. 3: Visualisierte Darstellung der Verdampferfahrwerte in CIM/21

\section{Meßtechnische Optimierung}

Die Auswertung von Meßergebnissen wurde wesentlich verbessert durch Verwendung von EXCEL. Die Rohdaten werden in ein Formular, welches unter EXCEL erstellt wurde, eingegeben und die Ergebnisse dann automatisch errechnet. Das Formular ist in Abb. 5 dargestellt.

Für die Auswertung der Rohdaten in EXCEL wurden folgende Umrechnungen einprogrammiert:

a) Druck

Der Absolutdruck errechnet sich aus dem angezeigten Manometerdruck unter Berücksichtigung des Barometerdruckes.

Absolutdruck=Manometeranzeigewert+Barometerdruck.

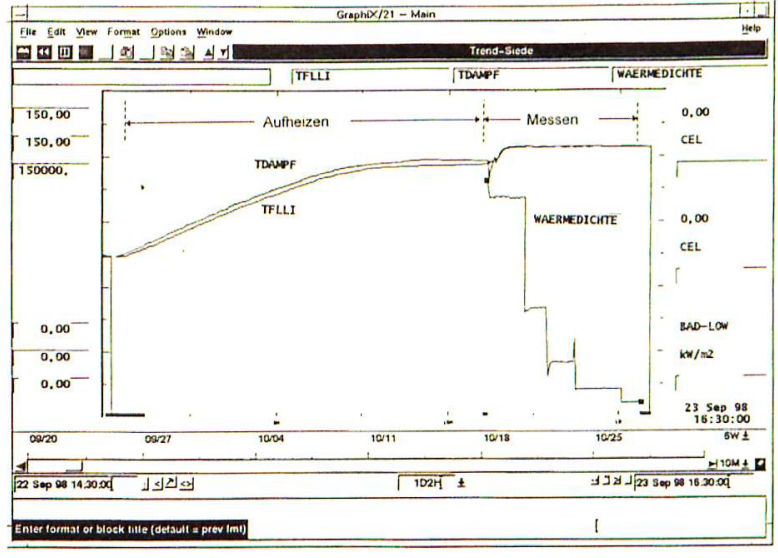

Abb. 4: Dyn. Verhalten $x=f(t)$ beispielhaft, d.h. Messgröße in Abhängigkeit von der Zeit für Dampftemperatur (TDAMPF), Flüssigkeitstemperatur (TFLLI) und Wärmestromdichte.

\section{b) Temperatur}

Die Thermospannungen werden auf Temperaturen umgerechnet mit der Formel für die Thermospannungskurve

$\mathrm{T}=3,8223 \cdot 10^{-11} \cdot \mathrm{U}^{3}-3,7597 \cdot 10^{-7} \cdot \mathrm{U}^{2}+2,5309 \cdot 10^{-2} \cdot \mathrm{U}+1,4452 \cdot 10^{-2} \quad$ (1) und im uibrigen wie in Teil I beschrieben [1] korrigiert. Die in Abb. 3 dargestellten Temperaturen werden mit PT 100 Temperaturfuihlern in 4 Leiter-Schaltung gemessen. Die Temperaturfuihler wurden mit einem Thermostat Typ Haake F6-C25 (Temperaturkonstanz $\pm 0,01 \mathrm{~K}$ ) bei $10^{\circ} \mathrm{C}$ und $80^{\circ} \mathrm{C}$ uiberprüft, wie in Tab. 1 dargestellt.

\begin{tabular}{l|l|l|l}
$\begin{array}{l}\text { Haake } \\
\text { Thermostat } /{ }^{\circ} \mathrm{C}\end{array}$ & TFLRE $/{ }^{\circ} \mathrm{C}$ & TFLLI $/{ }^{\circ} \mathrm{C}$ & TDAMPF $1 /{ }^{\circ} \mathrm{C}$ \\
\hline 10,00 & $10,03 \pm 0,05$ & $9,72 \pm 0,05$ & $9,82 \pm 0,2$ \\
\hline 80,00 & $79,65 \pm 0,1$ & $79,7 \pm 0,1$ & $79,9 \pm 0,1$ \\
\hline
\end{tabular}

Tab.1: Vergleich der PT100-Temperaturanzeigen mit einem Thermostat

\begin{tabular}{|c|c|c|c|c|c|c|c|c|c|c|}
\hline & Datum: & 18.09 .98 & Name: & Ignaszewski & \multicolumn{5}{|c|}{ Versuchsstoff: $19 \mathrm{~mol} \%$ Ethanol - $81 \mathrm{~mol} \%$ Wasser } & \\
\hline \multicolumn{5}{|c|}{ Barometer: } & \multicolumn{3}{|c|}{ Manometerkalibrierung: } & \multicolumn{2}{|c|}{ Messingrohr } & \\
\hline & & 1024 & [mbar] & & 0 bar $\wedge$ & 1019 & mbar & \multicolumn{2}{|c|}{$\lambda=100$} & \\
\hline & & \multicolumn{3}{|c|}{ 1. Messung } & \multicolumn{3}{|c|}{ 2. Messung } & \multicolumn{3}{|c|}{ 3.Messung } \\
\hline \multicolumn{2}{|c|}{ Uhrzeit } & \multicolumn{2}{|c|}{$14: 30$} & Absolutdruck & \multicolumn{2}{|l|}{$14: 45$} & Absolutdruck & \multicolumn{2}{|l|}{$15: 00$} & \\
\hline \multirow{2}{*}{\multicolumn{2}{|c|}{\begin{tabular}{|l|} 
Druck \\
Klimazelle
\end{tabular}}} & 5.00 & [bar] & \multirow{2}{*}{\begin{tabular}{rr}
\cline { 2 - 2 } & $5,005 \quad[\mathrm{bar}]$ \\
& $130,586\left[{ }^{\circ} \mathrm{C}\right]$
\end{tabular}} & 5,00 & [bar] & \multirow{2}{*}{$\begin{array}{r}5,005 \quad[\mathrm{bar}] \\
130,537\left[{ }^{\circ} \mathrm{C}\right]\end{array}$} & 5.00 & [bar] & $\begin{array}{l}\text { Absolutdruck } \\
5,005 \text { [bar] }\end{array}$ \\
\hline & zelle & 5,353 & {$[\mathrm{mV}]$} & & 5,351 & {$[\mathrm{mV}]$} & & 5,357 & {$[\mathrm{mV}]$} & $\therefore \quad 130,684\left[\left[^{\circ} \mathrm{C}\right]\right.$ \\
\hline \multicolumn{2}{|c|}{ Kühlkreislauf } & 126.9 & {$\left[{ }^{\circ} \mathrm{C}\right]$} & & 126,8 & {$\left[{ }^{\circ} \mathrm{C}\right]$} & \multirow[b]{2}{*}{$\begin{array}{ll}P & 35,69395[\mathrm{~W}]\end{array}$} & 126.8 & {$\left[{ }^{\circ} \mathrm{C}\right]$} & \\
\hline \multicolumn{2}{|c|}{ Spannung $\cup(2 x)$} & 4,343 & {$[\mathrm{~V}]$} & \multirow{2}{*}{$\begin{array}{ll}P & 35,716832[\mathrm{~W}] \\
\mathrm{q} & 6266.1109\left[\mathrm{~W} / \mathrm{m}^{2}\right]\end{array}$} & 4,336 & {$[\mathrm{~V}]$} & & 4,302 & {$[\mathrm{~V}]$} & \multirow{2}{*}{$\begin{array}{ll}P & 35,21617[\mathrm{~W}] \\
q & 6178,276\left[\mathrm{~W} / \mathrm{m}^{2}\right]\end{array}$} \\
\hline \multicolumn{2}{|c|}{ Strom I } & 4,112 & {$[\mathrm{~A}]$} & & 4,116 & {$[\mathrm{~A}]$} & $6262.097\left[\mathrm{~W} / \mathrm{m}^{2}\right]$ & 4.093 & {$[\mathrm{~A}]$} & \\
\hline Dampf & & 5,4130 & {$[\mathrm{mV}]$} & $\therefore \quad 132,061\left[{ }^{\circ} \mathrm{C}\right]$ & 5,4108 & {$[\mathrm{mV}]$} & $132,007\left[{ }^{\circ} \mathrm{C}\right]$ & 5,4097 & {$[\mathrm{mV}]$} & $\wedge \quad 131,980\left[{ }^{\circ} \mathrm{C}\right]$ \\
\hline Flüssi & igkeit 1(10) & 5,4480 & {$[\mathrm{mV}]$} & $\overline{T_{F !}}$ & 5,4470 & {$[\mathrm{mV}]$} & $\overline{T_{F I}}$ & 5,4449 & $\overline{[\mathrm{mV}]}$ & $\overline{T_{F 1}}$ \\
\hline & $2(11)$ & 5,4120 & {$[\mathrm{mV}]$} & $5,426[\mathrm{mV}]$ & 5,4090 & {$[\mathrm{mV}]$} & $5,424[\mathrm{mV}]$ & 5,4090 & {$[\mathrm{mV}]$} & $5,423[\mathrm{mV}]$ \\
\hline & $3(12)$ & 5,4180 & {$[\mathrm{mV}]$} & $132,381\left[{ }^{\circ} \mathrm{C}\right]$ & 5,4160 & {$[\mathrm{mV}]$} & $132,331\left[{ }^{\circ} \mathrm{C}\right]$ & 5.4150 & {$[\mathrm{mV}]$} & $\therefore \quad 132,306\left[^{\circ} \mathrm{C}\right]$ \\
\hline$\Delta T_{\text {Ront }}$ & Nullpunkt & Messung & korrigiert & $\Delta T_{\text {mittel }}$ & Messung & korrigiert & $\Delta T_{\text {mittei }}$ & Messung & korrigiert & $\Delta T_{\text {mittel }}$ \\
\hline $1(1)$ & 0,0085 & \begin{tabular}{|l|}
0,0871 \\
\end{tabular} & 0,0786 & $=\quad 0,0827[\mathrm{mV}]$ & \begin{tabular}{|l|}
0,0889 \\
\end{tabular} & 0,0804 & $=0,082457[\mathrm{mV}]$ & \begin{tabular}{|l|}
0,0889 \\
\end{tabular} & 0,0804 & $=0,0823[\mathrm{mV}]$ \\
\hline $2(2)$ & $-0,0048$ & 0,0722 & 0,0770 & $2,105[\mathrm{~K}]$ & 0,0742 & 0,0790 & $2.099[\mathrm{~K}]$ & 0,0749 & 0,0797 & $2.095[\mathrm{~K}]$ \\
\hline $3(4)$ & $-0,0002$ & 0,0791 & 0,0793 & & 0,0785 & 0,0787 & & 0,0782 & 0,0784 & \\
\hline $4(5)$ & 0,0056 & 0,0869 & 0,0813 & & 0,0857 & 0,0801 & & 0,0861 & 0,0805 & \\
\hline $5(6)$ & 0,0029 & 0,0919 & 0,0890 & $\Delta T_{\text {, korr }}$ & 0,0908 & 0,0879 & $\Delta \mathrm{T}_{\text {, korr }}$ & 0,0899 & 0,0870 & $\Delta T_{\text {chorr }}$ \\
\hline $6(7)$ & 0,0085 & 0.0951 & 0,0866 & $2,033[\mathrm{~K}]$ & 0,0938 & 0,0853 & $=2.027[\mathrm{~K}]$ & 0,0932 & 0.0847 & $=2.024[\mathrm{~K}]$ \\
\hline $7(8)$ & 0,0031 & 0,0902 & 0,0871 & & 0,0889 & 0,0858 & & 0,0885 & 0,0854 & \\
\hline & & & $3082,369[$ & {$\left[\mathrm{W} / \mathrm{m}^{2} \mathrm{~K}\right]$} & & 3089,644 & {$\left[\mathrm{~W} / \mathrm{m}^{2} \mathrm{~K}\right]$} & $\alpha=$ & 3052.811 & {$\left[\mathrm{~W} / \mathrm{m}^{2} \mathrm{~K}\right]$} \\
\hline & $q_{\text {mitel }}=$ & 6266.111 & {$\left[\mathrm{~W} / \mathrm{m}^{2}\right]$} & $\Delta T_{\text {mittel }}=$ & 2,033 & {$[\mathrm{~K}]$} & $\alpha_{\text {mittel }}=$ & 3082.369 [] & {$\left[\mathrm{W} / \mathrm{m}^{2} \mathrm{~K}\right]$} & \\
\hline
\end{tabular}

Abb. 5: Formular zur Auswertung eines Meßpunktes $(\triangle T, \dot{\mathrm{q}}, \alpha)$ in EXCEL. 
c) Wärmestromdichte

Die Wärmestromdichte wird aus der Leistung des Heizrohres, abziiglich des Spannungsabfalls ïber den kalten Enden des Heizdrahtes errechnet und bezogen auf die Rohroberfläche nach folgender Formel berechnet:

$$
\dot{\mathrm{q}}=\frac{\mathrm{I} \cdot \mathrm{U}}{0,005 \mathrm{~m}^{2}} \cdot\left(\frac{1}{\frac{560 \mathrm{~mm}}{200 \mathrm{~mm} \cdot 20}+1}\right)=0,877 \cdot \mathrm{U} \cdot \mathrm{I}\left[\frac{\mathrm{w}}{\mathrm{m}^{2}}\right]
$$

\section{d) Rohrwandüberhitzung}

Die Rohrwanduiberhitzung wird aus dem Meßwert, der Nullpunktspannung und Temperaturgefälle in der Heizrohrwand errechnet, nach folgenden Formeln:

$$
\mathrm{U}_{\text {korrigiert }}=\mathrm{U}_{\text {Messung }}-\mathrm{U}_{\text {Nullpunkt }}
$$

Die Temperaturdifferenz $\Delta \mathrm{T}$ wird aus $\mathrm{U}_{\text {eff }}$ nach Formel(1) berechnet und um den Temperaturabfall in der zylindrischen Wand korrigiert:

$$
\Delta \mathrm{T}=\Delta \mathrm{T}_{\mathrm{eff}}-\left(\dot{\mathrm{q}} \cdot 0,004 \cdot \frac{\ln \left(\frac{0,004}{0,003}\right)}{\lambda}\right)
$$

\section{e) Wärmeübergangskoeffizient}

Der Wärmeuibergangskoeffizient wird aus der Wärmestromdichte und der korrigierten Rohrwandiberhitzung wie folgt errechnet:

$$
\alpha=\frac{\dot{q}}{\Delta \mathrm{T}}
$$

\section{f) Mittelwerte}

Die Wärmestromdichte $\dot{\mathrm{q}}_{\text {mittel }}$, die Rohrwandüberhitzung $\Delta \mathrm{T}_{\text {mittel }}$ und Wärmeübergangskoeffizient $\alpha_{\text {mittel }}$ werden fuir einen Meßpunkt aus drei zeitlich aufeinanderfolgenden Einzelmessungen arithmetisch gemittelt.

\section{Kontrollmessungen}

Kontrollmessungen im Bereich des voll ausgebildeten Blasensiedens wurden bei 1 bar und bei 5 bar mit reinem Ethanol, vergälltem Ethanol, einer Ethanol/WasserMischung und einer Ethanol/Wasser/Aceton-Mischung durchgeführt.

\subsection{Reines Ethanol}

Wie in Abb. 5 ersichtlich, ist der Wärmeübergangskoeffizient für reines Ethanol bei gleicher Wärmestromdichte erwartungsgemäß höher als für vergälltes Ethanol sowohl bei 1 bar wie auch bei 5 bar. So z.B. ist der WÜK für reines Ethanol und $\dot{q}=4 \mathrm{~kW}$ bei $p=1$ bar um $65 \%$ und bei $p=5$ bar um 26\% höher als für vergälltes Ethanol. Die Steigungen der Ausgleichsgeraden sind bei gleichem Druck fuir reines und vergälltes Ethanol annähernd gleich. (Tab.2).

\begin{tabular}{l|l|l} 
& Ethanol & Ethanol mit $1 \mathrm{~mol} \%$ MEK \\
\hline $\mathbf{1}$ bar & $\mathrm{n}=0,822$ & $\mathrm{n}=0,841$ \\
\hline $\mathbf{5}$ bar & $\mathrm{n}=0,732$ & $\mathrm{n}=0,738$
\end{tabular}

Tab. 2: Steigungen der Ausgleichsgeraden für reines und vergälltes Ethanol

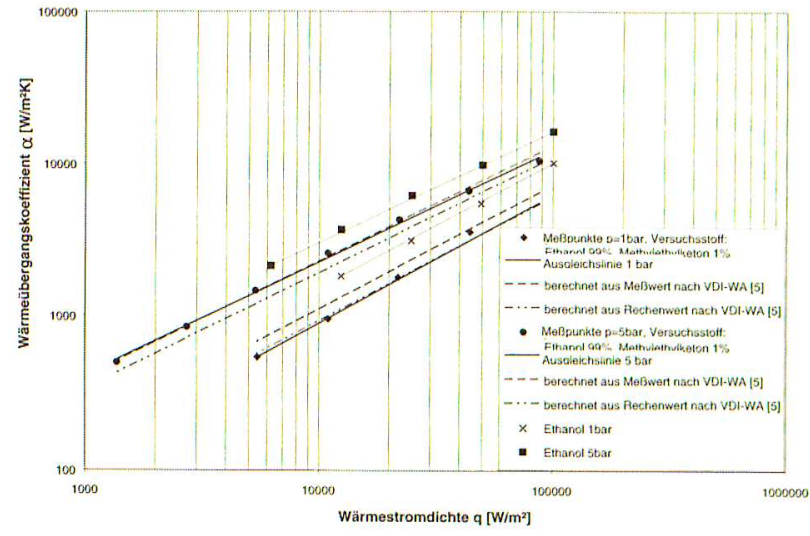

Abb.6: Wärmeübergangskoeffizient $\alpha$ in Abhängigkeit von der Wärmestromdichte $\dot{\mathrm{q}}$ bei 1 und 5 bar für vergälltes und reines Ethanol.

\subsection{Ethanol/ Wasser-Mischung bei 1 bar}

Es wurde eine Ethanol/Wasser-Mischung hergestellt, für die sich gaschromatographisch eine mittlere Zusammensetzung der Flüssigkeit von $\mathrm{x}_{\mathrm{ETOH}}=0,185$ ergab. Weitere Angaben zur Analytik sind in Kapitel 4 und in Teil III dieser Arbeit enthalten. Für die Mischung Ethanol/Wasser wurden Messungen bei zwei Drücken, 1 bar und 5bar, und jeweils 5 Wärmestromdichten im Bereich zwischen 6 bis $100 \frac{\mathrm{kW}}{\mathrm{m}^{2}}$ durchgeführt.

\subsubsection{Messungen bei 1 bar und $100 \mathrm{~kW} / \mathrm{m}^{2}$}

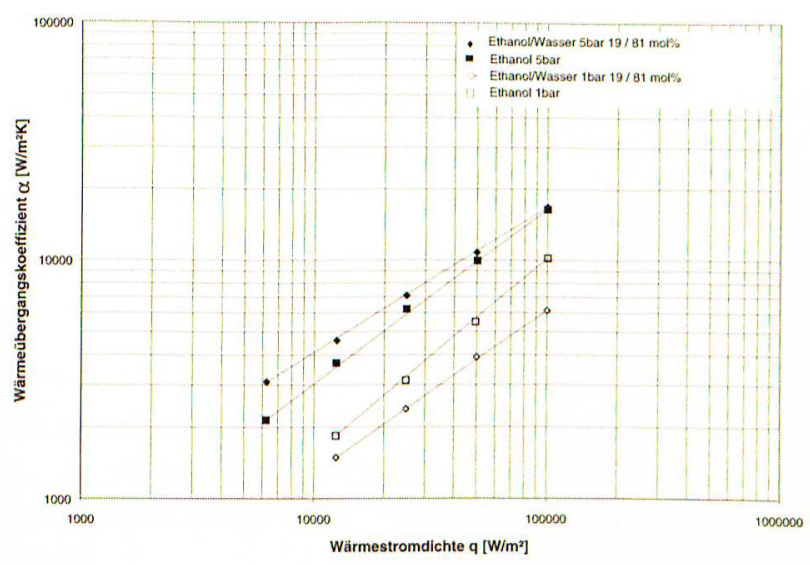

Abb. 7: Wärmeübergangskoeffizient $\alpha$ in Abhängigkeit von der Wärmestromdichte $\dot{\mathrm{q}}$ für reines Ethanol und Ethanol/Wasser-Gemisch bei 1 und 5 bar

Wie erwartet und im Einklang mit der Literatur zeigte sich, daß der Wärmeübergangskoeffizient $\alpha_{\text {Mix }}$ der Mischung niedriger als $\alpha$ des reinen Ethanols ist. Die relative Verschlechterung des Wärmeübergangs aufgrund des „Gemischeffektes“ beträgt demnach für $100 \mathrm{~kW} / \mathrm{m}^{2}$ $39 \%$ und für $20 \mathrm{~kW} / \mathrm{m}^{2} 26 \%$.

Die Steigungen der Ausgleichsgerade für das Ethanol/ Wasser-Gemisch ist erwartungsgemäß kleiner als für reines Ethanol: $\mathrm{n}_{\mathrm{Mix}}=0,68<\mathrm{n}_{\mathrm{Eth}}=0,82$.

Zur Ermittlung des molanteilig gewichteten, mittleren Wärmeübergangskoeffizienten $\alpha_{\text {id }}$ für die Mischung wird folgende Formel verwendet:

$\alpha_{\text {id }}=\bar{x}_{1} \cdot \alpha_{1}+\bar{x}_{2} \cdot \alpha_{2}$

Bei einer Wärmestromdichte von $\dot{\mathrm{q}}=100 \frac{\mathrm{kW}}{\mathrm{m}^{2} \mathrm{~K}}$ ergibt sich mit den Reinstoffwerten $\alpha_{\text {Ehanol }}=10000 \underset{\mathrm{m}^{2} \mathrm{~K}}{\mathrm{w}}, \alpha_{\text {Wasser }}=9500 \frac{\mathrm{w}}{\mathrm{m}^{2} \mathrm{~K}}$ [ 9 ] ein molanteiliger Mittelwert von $\alpha_{\text {id }}=9592 \frac{\mathrm{w}}{\mathrm{m}^{2} \mathrm{~K}}$. $\frac{\alpha_{\text {Mix }}}{\alpha_{\text {id }}}=\frac{6100 \mathrm{~W} / \mathrm{m}^{2} \mathrm{~K}}{9592 \mathrm{~W} / \mathrm{m}^{2} \mathrm{~K}}=\underline{\underline{0,636}}$ 


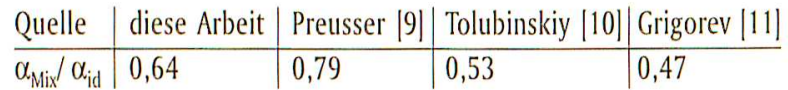
$\dot{\mathrm{q}}=100 \mathrm{~kW} / \mathrm{m}^{2}$ und $\mathrm{p}=1 \mathrm{bar}$

Tab. 3: Verhältnis $\alpha_{\text {Mix }} \alpha_{i d}$,errechnet aus den Messungen verschiedener Autoren, im Vergleich

Wie Tab. 3 zeigt, weichen die von verschieden Autoren gemessenen Werte der relativen Verschlechterung stark voneinander ab. Die eigenen Meßwerte nehmen dabei eine Mittelstellung ein. Möglicherweise ist die Streuung der Ergebnisse verschiedener Autoren auch dadurch zu erklären, daß sich während der Messung korrosionsbedingte Veränderungen der Oberflächenrauigkeit häufig dann ergeben, wenn eine der Komponenten Wasser ist. (siehe auch Messungen für Ethanol+Wasser bei $5 \mathrm{bar}$ und Ethanol+ Wasser+Aceton)

\subsubsection{Messungen bei 1 bar und $20 \mathrm{~kW} / \mathrm{m}^{2}$}

Für die Wärmestromdichte von $20 \frac{\mathrm{kW}}{\mathrm{m}^{2}}$ wurde $\alpha_{\text {Wasser }}$ aus Literaturwerten ermittelt, da eigene Messungen mit reinem Wasser fehlen.

Der $\alpha$-Wert wurde auf zwei Wegen abgeschätzt: Aus dem Wert $\alpha=6000 \frac{\mathrm{w}}{\mathrm{m}^{2} \mathrm{~K}}$ gemäß VDI-Wärmeatlas Ha 11 [7] ergibt sich durch Umrechnen von $p^{*}=0,1$ auf $p^{*}=1 / 220,9$ bei konstanter Wärmestromdichte mit der Formel

$\mathrm{F}\left(\mathrm{p}^{* \prime}\right)=1,73 \mathrm{p}^{* 0,27}+\left[6,1+\frac{0,68}{1-\mathrm{p}^{* 2}}\right] \mathrm{p}^{* 2}$

und $\mathrm{n}=0,9-0,3 \mathrm{p}^{\mathrm{*} 0,15}$

\section{ein Wert von $\alpha=2414 \mathrm{~W} /\left(\mathrm{m}^{2} \mathrm{~K}\right)$}

Aus dem von Preusser bei $\dot{q}=100 \underset{\mathrm{m}^{2}}{\mathrm{kw}}$ und $\mathrm{p}=1$ bar angegebenen Wert von $\alpha=9500 \underset{m^{2} \mathrm{~K}}{\mathrm{w}}$ [3] ergab sich durch Umrechnung von $\dot{\mathrm{q}}=100 \frac{\mathrm{kW}}{\mathrm{m}^{2}}$ auf $\dot{\mathrm{q}}=20 \frac{\mathrm{kW}}{\mathrm{m}^{2}}$ bei konstantem Druck ein Wert von $\alpha=2767 \underset{m^{2} \mathrm{~K}}{\mathrm{~W}}$. Die Abweichung zwischen den beiden Werten ist mit $12,8 \%$ relativ akzeptabel, es wurde aber hier der umgerechnete PreusserWert verwendet.

Das Verhältnis $\alpha_{\text {Mix }} / \alpha_{\text {id }}$ bei der Wärmestromdichte von $20 \frac{\mathrm{kW}}{\mathrm{m}^{2}}$, ergibt sich aus $\alpha_{\text {Ethanol }}=2700 \frac{\mathrm{w}}{\mathrm{m}^{2} \mathrm{~K}}$, dem errechneten $\alpha_{\text {Wasser }}=2767 \frac{\mathrm{w}}{\mathrm{m}^{2} \mathrm{~K}}$ sowie aus dem molanteiligen Mittelwert $\alpha_{\mathrm{id}}=2754 \frac{\mathrm{w}}{\mathrm{m}^{2} \mathrm{~K}}$

$\frac{\alpha_{\text {Mix }}}{\alpha_{\text {id }}}=\frac{2000 \mathrm{~W} / \mathrm{m}^{2} \mathrm{~K}}{2754 \mathrm{~W} / \mathrm{m}^{2} \mathrm{~K}}=\underline{\underline{0,726}}$

Wie erwartet ist die relative Verschlechterung des Wärmestoffuibergangs bei der kleineren Wärmestromdichte weniger stark ausgeprägt.

\subsection{Ethanol/Wasser-Gemisch bei 5 bar}

Wider Erwarten zeigte sich, daß der Wärmeübergangskoeffizient $\alpha_{\text {Mix }}$ der Mischung höher als $\alpha$ des reinen Ethanols ist. Die relative Verbesserung des Wärmeübergangs aufgrund des „Gemischeffektes“ beträgt demnach für $20 \mathrm{~kW} / \mathrm{m}^{2} 20 \%$, bei $100 \mathrm{~kW} / \mathrm{m}^{2}$ ist der Wärmeüber- gangskoeffizient nahezu gleich groß wie für die reinen Komponenten. Dies kann durch eine Veränderung der Rohroberfläche geschehen sein und läßt sich aufgrund der Farbänderung des Rohres vermuten. Durch Änderung der Rauhigkeit kann die Anzahl der Keimstellen zur Blasenbildung erhöht sein und somit eine Verbesserung des Wärmeuibergangs eintreten, siehe auch Kapitel 3.2.1.

Die Steigungen der Ausgleichsgerade für das Ethanol/ Wasser-Gemisch ist erwartungsgemäß kleiner als für reines Ethanol

$\mathrm{n}_{\mathrm{Mix}}=0,61<\mathrm{n}_{\mathrm{Eth}}=0,73$.

\subsection{Ethanol/Wasser/Aceton bei 1 bar}

Wie erwartet und im Einklang mit der Literatur zeigte sich, daß auch der Wärmeüibergangskoeffizient $\alpha_{\text {Mix }}$ der ternären Mischung niedriger als $\alpha$ des reinen Ethanols ist. Die relative Verschlechterung des Wärmeübergangs aufgrund des „Gemischeffektes“ beträgt demnach für $100 \mathrm{~kW} / \mathrm{m}^{2} 53 \%$ und für $20 \mathrm{~kW} / \mathrm{m}^{2} 15 \%$ im Vergleich zum reinen Ethanol.

Die Steigungen der Ausgleichsgerade für das Ethanol/ Wasser/Aceton-Gemisch ist erwartungsgemäß kleiner als für reines Ethanol:

$\mathrm{n}_{\text {tern }}=0,54<\mathrm{n}_{\text {bin }}=0,68<\mathrm{n}_{\mathrm{Eth}}=0,82$.

Im Vergleich zu dem binären Gemisch nimmt die Verschlechterung des WÜ erwartungsgemäß zu, der Effekt verschwindet aber bei kleineren Wärmestromdichten $\dot{\mathrm{q}}<20 \frac{\mathrm{kW}}{\mathrm{m}^{2}}$

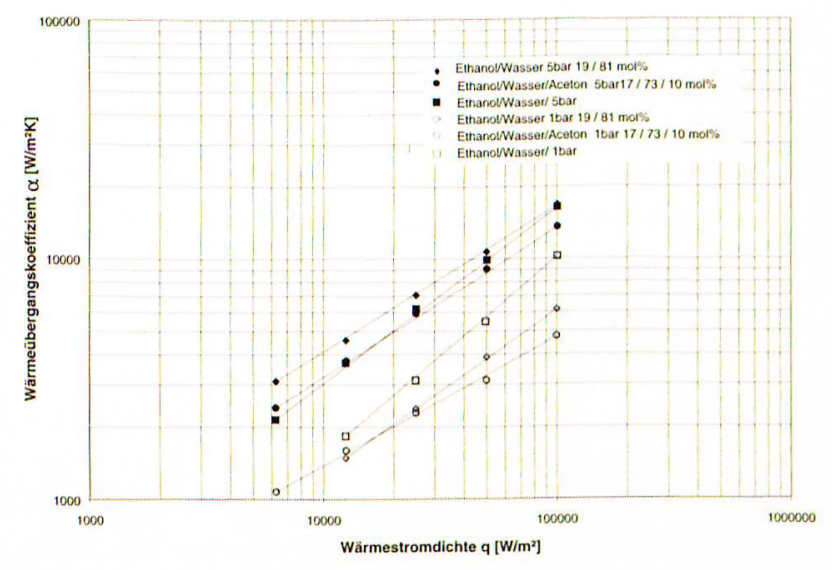

Abb. 8: Wärmeübergangskoeffizient $\alpha$ in Abhängigkeit von der Wärmestromdichte $\dot{\mathrm{q}}$ für Ethanol, Ethanol/Wasser-Mischung und Ethanol/Wasser/AcetonMischung

\subsection{Ethanol/ Wasser/ Aceton bei 5bar}

Die relative Verschlechterung des Wärmeübergangs aufgrund des „Gemischeffektes“ beträgt fuir $100 \mathrm{~kW} / \mathrm{m}^{2}$ $18 \%$ im Vergleich zu reinem Ethanol und verschwindet bei etwa für $20 \mathrm{~kW} / \mathrm{m}^{2}$, wie aus Abb. 7 hervorgeht. Die Steigungen der Ausgleichsgerade für das Ethanol/ Wasser/Aceton-Gemisch ist erwartungsgemäß kleiner als fuir reines Ethanol: $n_{\text {tern }}=0,62<n_{E \text { th }}=0,73$ Aufschlußreicher ist aber der Vergleich mit dem binären Gemisch aus dem in Kap. 3.3 genannten Grund, $\mathrm{n}_{\text {tern }}$ ist demnach vergleichbar mit $n_{\text {bin }}$.

Vergleich der $\alpha_{\text {Werte }}$ bei

$\dot{\mathrm{q}}=10 \frac{\mathrm{kW}}{\mathrm{m}^{2}}: \alpha_{\text {tern }}=3300 \underset{\mathrm{m}^{2} \mathrm{~K}}{\mathrm{w}}, \alpha_{\text {bin }}=4200 \frac{\mathrm{w}}{\mathrm{m}^{2} \mathrm{k}}$ 
Erwartungsgemäß ist der Wärmeuibergangskoeffizient für die ternäre Mischung geringer als für die binäre Mischung und die reinen Komponenten.

\section{Gaschromatographische Analytik}

Für die Konzentrationsmengen wurde ein Gas-Chromatograph Typ Chrompack CP 9000 mit bepackter Kolonne und Wärmeleitsfähigkeitsdetektor eingesetzt. Angaben zu Kalibrierungen, Messgenauigkeiten und Meßergebnissen sind in Teil III dieser Arbeit enthalten. Parallel zu den Wärmeübergangsmessungen wurden Proben aus dem Flüssigkeitspool des Verdampfers bei jedem Beharrungszustand konstanter Wärmestromdichte entnommen und gaschromatographisch untersucht. Die Ergebnisse der Konzentrationsmessungen sind in den Abb. 9,10 und 11 für die binäre Mischung Ethanol/Wasser und für die ternäre Mischung Aceton-Ethanol-Wasser enthalten.

Erwartungsgemäß ergab sich bei keiner Messreihe der untersuchten Gemische eine signifikante Abhängigkeit der Flüssigkeitskonzentration von der Wärmestromdichte. Die Schwankungen um den Mittelwert sind kleiner als $1 \mathrm{~mol} \%$ und liegen damit in der Größenordnung früherer Messungen [3]. Die teilweise größeren Abweichungen in Abb. 9 sind eine Folge von nicht ausreichend stabilen Beharrungszuständen bei den ersten Probenmessungen.

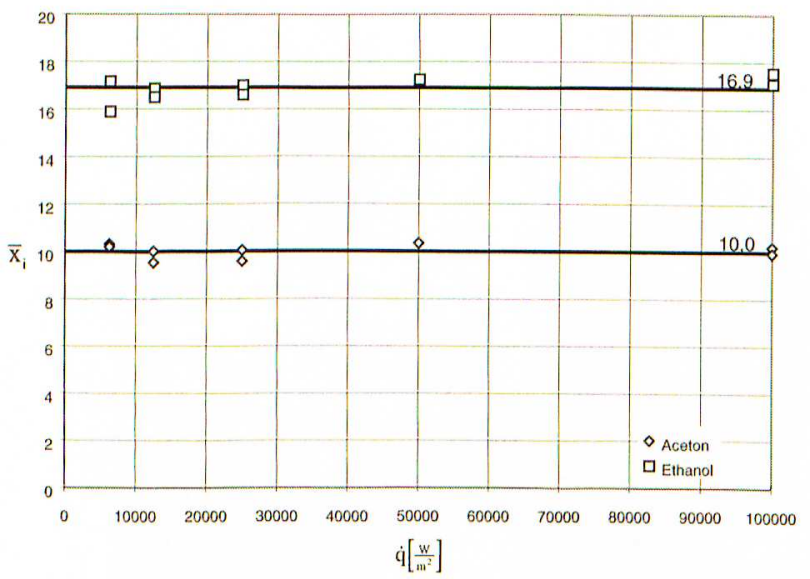

Abb. 9: Abhängigkeit des Molenbruchs $\overline{\mathrm{X}}_{\mathrm{i}}$ von der Wärmestromdichte $\dot{\mathrm{q}}$ für das ternäre Stoffsystem Aceton(1)-Ethanol(2)-Wasser(3) bei $p=1$ bar

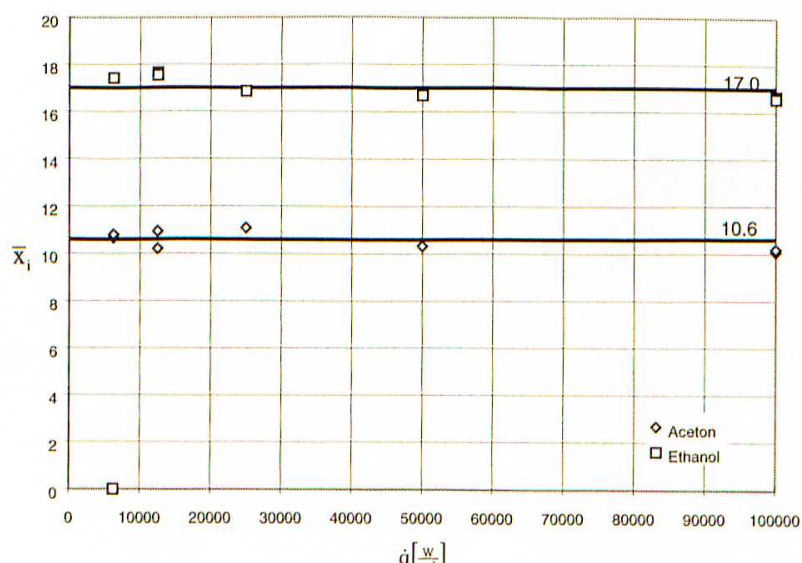

Abb. 10: Abhängigkeit des Molenbrichs $\overline{\mathrm{X}}_{\mathrm{i}}$ von der Wärmestromdichte $\dot{\mathrm{q}}$ für das ternäre Stoffsystem Aceton(1)-Ethanol(2)-Wasser(3) bei $p=5$ bar

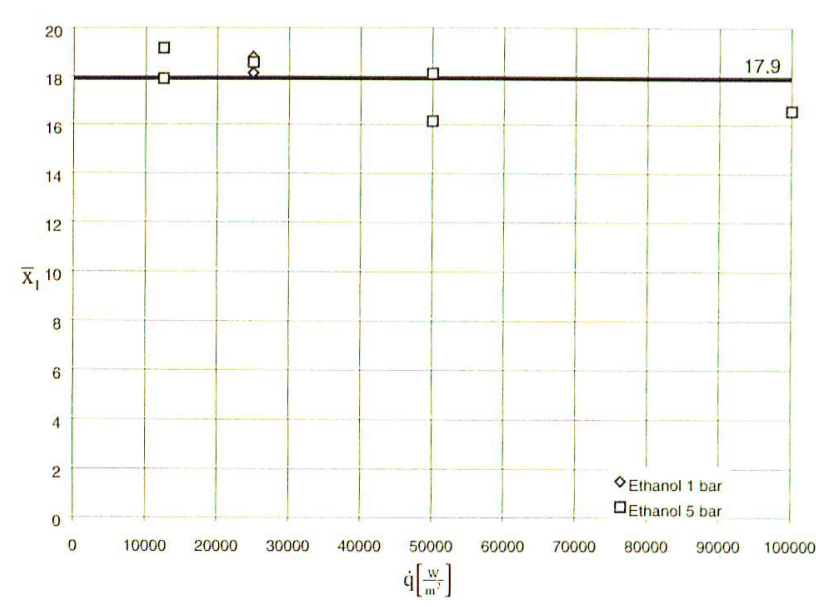

Abb. 11: Abhängigkeit des Ethanol-Molenbruchs $\overline{\mathrm{X}}_{1}$ von der Wärmestromdichte $\dot{q}$ für das binäre Stoffsystem Ethanol(1)-Wasser(1) bei $p=1$ und 5 bar

\section{Zusammenfassung}

In dieser Arbeit wurde eine für Wärmeübergangsmessungen an reinen Stoffen aufgebaute Präzisionsapparatur fuir Untersuchungen an Mehrstoffsystemen umgerïstet und weitgehend automatisiert. Erste Kontrollmessungen lieferten Ergebnisse, die mit Literaturangaben gut harmonieren. Entwicklungsbedarf besteht für die Zukunft hinsichtlich

- der Optimierung der Dampf-Probenahme

- der weiteren Automatisierung der Meßwerteerfassung und

- der weiteren Untersuchung insbesondere wässriger Mehrstoffsysteme.

Danksagung: Dank gebührt vor allem Herrn em. Prof. Dr. Bier $\dagger$ (Universität Karlsruhe) für die uns geschenkte Apparatur.

Für die finanzielle Unterstiitzung dieses Forschungsvorhabens sind wir dem Bundesministerium für Bildung, Wissenschaft, Forschung und Technologie zu Dank verpflichtet.

\section{Literatur}

[1] Schmadl, J. und Ignatschewski,P.:"Inbetriebnahme einer Präzisionsapparatur für Wärmeübergangsmessungen beim Sieden reiner Stoffe und Nachrüstung für Stoffgemische/Teil I“, wissenschaftliche Beiträge, TFH Wildau, Heft 2/1998

[2] Schmadl, J.: "Prozeßleitsystem mit Rechnereinsatz für die Überwachung, Steuerung, und Berechnung thermischer Trennprozesse, Apparate und Anlagen", Wissenschaftliche Beiträge TFH- Wildau, Heft1/1995

[3] Schmadl, J.: Wärmeübertragung beim Blasensieden binärer Stoffgemische unter hohem Druck, Dissertation Universität Karlsruhe, 1982.

[4] Goetz. J.: Entwicklung und Erprobung einer Normapparatur zur Messung des Wärmeübergangs beim Blasensieden, Dissertation Universität Karlsruhe, 1980. 
[5] Bonekamp, S.: Einfluß von Ultraschall auf den Wärmeübergang beim Blasensieden binärer Gemische, Dissertation Universität Karlsruhe, 1997.

[6] Gorenflo, D.: Zum Wärmeübergang bei der Blasenverdampfung an Rippenrohren, Dissertation Technische Hochschule Karlsruhe, 1966.

[7] VDI-Wärmeatlas, 7. Auflage VDI-Verlag Duisseldorf, 1994.

[8] Onken, U. und Arlt, W.: Recommended Text Mixtures for Destillation Colums, sec. edition, the Institution of Chemical Engineers, G.E. Davis Bulding, Warwickshire.

[9] Preusser, P.: Wärmeübergang beim Verdampfen binärer und ternärer Flüssigkeitsgemische, Dissertation RuhrUniversität Bochum, 1978.

[10] Tolubinsky, V. I., Kroveshko, A. A, Ostrovskiy, Yu. N. und Pisarev, V. Ye: Effect of Pressure on Boiling Heat Transfer Rate in Water-Alcohol Mixtures, Heat Transfer-Soviet Research 5 (3), S. 66-68, 1973.

[11] Grigorev, L. N. und Usmanov, A. G.: Heat Transfer During Boiling of Binary Mixtures, Soviet Phys.-Techn. Phys. 3 (2), S. 297-305, 1958 .

\section{Autoren}

Prof. Dr.-Ing. Josef Schmadl

Dipl.-Ing. (FH) Peter Ignaszewski

Dipl.-Ing. Jens-Uwe Gerking

Technische Fachhochschule Wildau

Technikum für Thermische Verfahrenstechnik, Haus 8

Bahnhofstraße

15745 Wildau

Tel. (0 33 75) 508-110

Fax (0 30) 6757754

E-mail: jschmadl@igw.tfh-wildau.de 